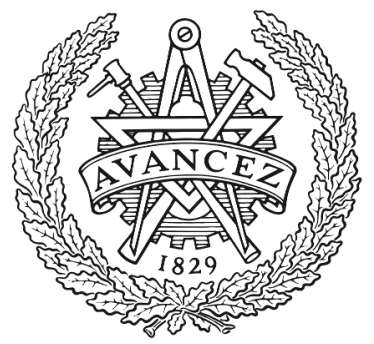

CHALMERS

UNIVERSITY OF TECHNOLOGY

\title{
Tuning the Thermoresponsive Behavior of Surface-Attached PNIPAM Networks: Varying the Crosslinker Content in SI-ATRP
}

Downloaded from: https://research.chalmers.se, 2023-04-26 11:38 UTC

Citation for the original published paper (version of record):

Thiele, S., Andersson, J., Dahlin, A. et al (2021). Tuning the Thermoresponsive Behavior of Surface-Attached PNIPAM Networks: Varying the

Crosslinker Content in SI-ATRP. Langmuir, 37(11): 3391-3398.

http://dx.doi.org/10.1021/acs.langmuir.0c03545

N.B. When citing this work, cite the original published paper. 


\title{
Tuning the Thermoresponsive Behavior of Surface-Attached PNIPAM Networks: Varying the Crosslinker Content in SI-ATRP
}

\author{
Sophia Thiele, John Andersson, Andreas Dahlin, and Rebekah L. N. Hailes*
}

Cite This: Langmuir 2021, 37, 3391-3398

Read Online

ABSTRACT: The synthesis and thermoresponsive properties of surface-attached poly $(N$ isopropylacrylamide)-co- $N, N^{\prime}$-methylene bisacrylamide (PNIPAM-co-MBAM) networks are investigated. The networks are formed via SI-ARGET-ATRP ("grafting-from") on thiolbased initiator-functionalized gold films. This method is reliable, well controlled, fast, and applicable to patterned surfaces (e.g., nanopores) for networks with dry thicknesses $>20$ $\mathrm{nm}$. Surface-attached PNIPAM-co-MBAM gels are swollen below their volume phase transition temperature but above collapse without complete expulsion of water (retain $\sim 50$ vol \%). The swelling/collapse transition is studied using complementary SPR and QCMD techniques. The ratio between swollen and collapsed heights characterizes the thermoresponsive behavior and is shown to not depend on network height but to vary with MBAM content. The higher the proportion of the crosslinker, the lower the magnitude of the phase transition, until all responsiveness is lost at $5 \mathrm{~mol} \%$ MBAM. The temperature range of the transition is broadened for more crosslinked PNIPAM-co-MBAM gels but remains centered around $32{ }^{\circ} \mathrm{C}$. Upon reswelling, less crosslinked networks display sharp transitions, while for those containing $\geq 3 \mathrm{~mol} \% \mathrm{MBAM}$, transitions remain broad. This tunable behavior persists for gels on nanostructured gold surfaces. Investigating PNIPAM-co-MBAM networks on gold plasmonic nanowell arrays is a starting point for expanding their scope as thermo-controlled nanoactuators.

\section{INTRODUCTION}

Poly (N-isopropyl acrylamide) (PNIPAM) is a particularly well-studied thermoresponsive polymer due to its biocompatibility and biologically relevant lower critical solution temperature (LCST) of $\sim 32{ }^{\circ} \mathrm{C}$ in aqueous media (i.e., close to physiological temperature). ${ }^{1,2}$ Above this critical solution temperature, the unfavorable entropic contribution from the hydrophobic effect to the free energy of mixing dominates over exothermic hydrogen bonding, decreasing the solvent quality of water to the point where the polymer undergoes a sharp transition from a hydrated, extended coil conformation to a hydrophobic, collapsed structure (which maximizes intra- and interchain interactions). By exploiting these responsive properties, PNIPAM hydrogels have been used in drug delivery systems, ${ }^{3}$ cell culturing, ${ }^{4}$ and artificial muscles. ${ }^{5}$ PNIPAM brushes, formed by end-tethered chains on a variety of surfaces, have served as thermo-controlled nanoactuators (such as valves, ${ }^{6}$ pumps, ${ }^{7}$ and filters $\left.{ }^{8}\right)$ in microfluidic devices. ${ }^{9,10}$ The extent of the thermally induced collapse of the polymer brushes depends on grafting density and molecular weight, ${ }^{11,12}$ both of which can be controlled by surface-initiated (also termed "grafting-from") atom transfer radical polymerization (SI-ATRP). ${ }^{13-22}$ The LCST is known to remain essentially the same as for coils in solution $\left( \pm 1^{\circ} \mathrm{C}\right)$ regardless of the grafting strategy.

The magnitude of the thermoresponsive transition can be controlled by introducing crosslinks between polymer chains.
For nonresponsive gels on substrates, crosslinkers significantly diminish the extent to which networks can swell. ${ }^{23-25}$ This has been exploited to vary the mechanical and swelling properties of poly(acrylamide) (PAAM) ${ }^{24}$ and furthered to include $\mathrm{pH}$ responsive polyelectrolyte brushes. ${ }^{26}$ Investigations into crosslinking PNIPAM brushes grafted from nanoparticles noted the change in volume phase transition temperature (VPTT), network mesh size, and permeability. ${ }^{27}$ To more comprehensively study the effect of crosslinker content on swelling behavior, Harmon et al. prepared PNIPAM gels via common free radical polymerizations prior to spin-coating on silica substrates. ${ }^{28}$ However, swelling factors varied even within samples containing the same proportion of crosslinker. This was thought to occur due to stress-induced orientation introduced to the PNIPAM networks in the spin-coating process. Additionally, surface-attached networks prepared via free radical polymerizations have been shown to have an inhomogeneous crosslinking density, especially compared to gels synthesized via a controlled/living radical polymerization

Received: December 14, 2020

Revised: February 24, 2021

Published: March 15, 2021 
Scheme 1. Chemical Structure of the ATRP Initiator on Gold, Reacting with PNIPAM and the Crosslinker, $N, N^{\prime}$ Methylenebisacrylamide, to Give Brush Networks
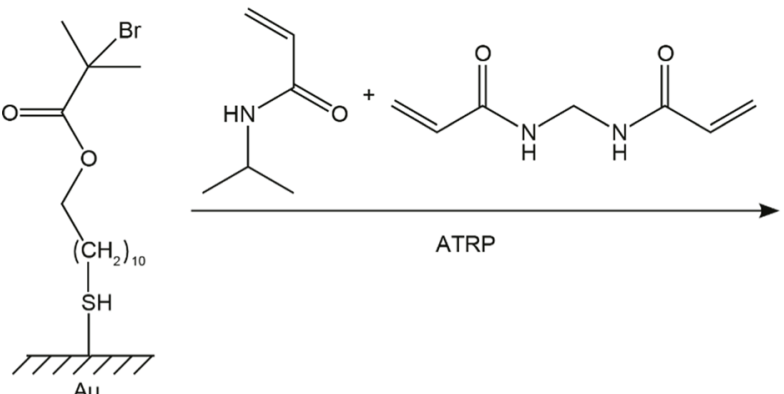

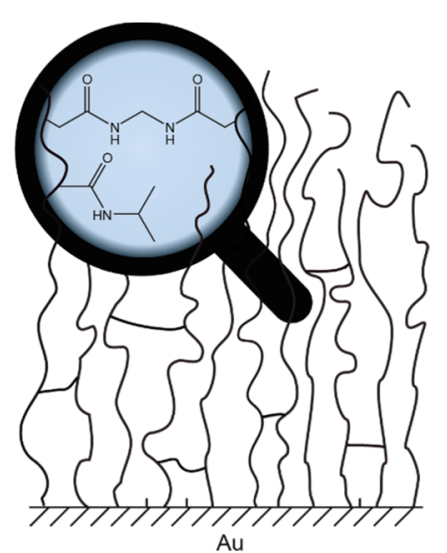

$\mathrm{Au}$ techniques, for example, ATRP. ${ }^{29}$ Besides homogeneous crosslinking, ATRP provides further advantages in surface functionalization: the transfer agents used are commercially available and show a high functional group tolerance, and the reaction is applicable to various surfaces (patterned or smooth) by choosing appropriate initiators. ${ }^{20}$ To circumvent limits imposed by the oxygen-sensitive nature of ATRP, a more tolerant option dubbed "activators regenerated via electron transfer" ATRP (ARGET-ATRP) is often used. ${ }^{30,31}$ To the best of our knowledge, there are no studies on the influence of crosslinking on the swelling/collapsing behavior of PNIPAM brushes, partly as determining the accurate brush heights in the solution is challenging. ${ }^{32,33}$ It should be noted that the degree of swelling is expected to depend on the geometry and orientation of the chains. For instance, in a brush on a planar surface (compared to a gel in $3 \mathrm{D}$ ), there is only one spatial dimension available for expansion.

In this work, we varied the amount of a covalent crosslinker, $N, N^{\prime}$-methylene bisacrylamide (MBAM), and reported its influence on the thermoresponsive behavior of PNIPAM brushes prepared via surface-initiated ARGET-ATRP (SIARGET-ATRP) on planar and patterned gold surfaces. Taking advantage of the metallic support, heights in the solution above and below the characteristic VPTT were obtained from SPR measurements using our previously reported non-interacting probe method. ${ }^{34}$ Quartz crystal microbalance with dissipation monitoring (QCMD) experiments support these results and give further insights into the phase transition temperature range. We discuss our results in the context of physisorbed and spin-coated PNIPAM-co-MBAM gels of similar thicknesses, ${ }^{28,35,36}$ PNIPAM networks on nanoparticles, ${ }^{27}$ and other types of crosslinked polymer brushes grafted from planar substrates via ATRP. ${ }^{24,25}$

\section{EXPERIMENTAL SECTION}

Chemicals. All chemicals were purchased from Sigma-Aldrich and used as received unless stated otherwise. $\mathrm{H}_{2} \mathrm{O}_{2}(30 \%)$ was from ACROS, $\mathrm{N}$-isopropyl acrylamide (NIPAM) and $\mathrm{NH}_{4} \mathrm{OH}(28-30 \%)$ from Fischer, $\mathrm{H}_{2} \mathrm{SO}_{4}$ (98\%) and EtOH (99.5\%) from SOLVECO, and $\omega$-mercaptoundecyl bromoisobutyrate from ProChimia. Water was of ASTM research grade type 1 ultrafiltered water (Milli-Q water). Buffers were based on phosphate-buffered saline (PBS) tablets (0.01 M phosphate, $0.13 \mathrm{M} \mathrm{NaCl}, \mathrm{pH} 7.4)$.

Monomer NIPAM (99\%) was recrystallized from hexane at $85{ }^{\circ} \mathrm{C}$ and stored under $\mathrm{N}_{2}$. The polymerization solvent $\mathrm{MeOH}$ was dried over $3 \AA$ molecular sieves (Merck) and then stored under $\mathrm{N}_{2}$.
Surface Cleaning. Prior to surface functionalization, SPR and QCMD sensors were cleaned in piranha wash $\left(\mathrm{H}_{2} \mathrm{SO}_{4} / \mathrm{H}_{2} \mathrm{O}_{2}, 3: 1 \mathrm{v} /\right.$ v) for $30 \mathrm{~min}$ and then rinsed in Milli-Q. The sensors and nanowellpatterned surfaces were then cleaned in RCAl wash $\left(\mathrm{H}_{2} \mathrm{O} / \mathrm{H}_{2} \mathrm{O}_{2} /\right.$ $\mathrm{NH}_{4} \mathrm{OH}$ 5:1:1 v/v) at $80{ }^{\circ} \mathrm{C}$ for $30 \mathrm{~min}$, rinsed in Milli-Q and EtOH, and then dried with $\mathrm{N}_{2}$.

SAM Formation. Clean gold surfaces were immersed in a $3 \mathrm{~mL}$ EtOH solution containing the ATRP initiator $\omega$-mercaptoundecyl bromoisobutyrate $(3 \mu \mathrm{L}, 2 \mathrm{mM})$ and shaken $(50 \mathrm{rpm})$ for $18 \mathrm{~h}$. After incubation, the substrates were rinsed in $\mathrm{EtOH}$ and dried under $\mathrm{N}_{2}$.

SI-ARGET-ATRP. PNIPAM brushes and PNIPAM-co-MBAM gels were prepared under the same reaction conditions. Reactions were carried out using standard Schlenk line techniques under an inert atmosphere of $\mathrm{N}_{2}$. The amount of MBAM supplied in the monomer feed varied between $0 \mathrm{~mol} \%$ (for brushes) up to $10 \mathrm{~mol} \%$. Depending on the desired crosslinker content, varying amounts of both monomers were used so that the total monomer concentration was always $0.96 \mathrm{M}$. An example synthesis of PNIPAM-co-MBAM $1 \%$ is described below.

In one flask, monomer NIPAM (1.076 g, 9.5 mmol, 792 equiv), crosslinker MBAM (14.8 mg, $0.096 \mathrm{mmol}, 8$ equiv), and $\mathrm{MeOH}$ (8 $\mathrm{mL}$ ) were added to the inhibitor remover. The solution was degassed with $\mathrm{N}_{2}$ for $5 \mathrm{~min}$. In a second flask, $\mathrm{CuBr}_{2}(2.7 \mathrm{mg}, 0.012 \mathrm{mmol}, 1$ equiv) was added to PMDETA ( $26.7 \mu \mathrm{L}, 0.128 \mathrm{mmol}, 10.7$ equiv). The monomer solution was filtered $(0.2 \mu \mathrm{m}$ PTFE syringe filter) into the second flask, and the light blue solution obtained was degassed for a further $20 \mathrm{~min}$. Separately, ascorbic acid $(8.5 \mathrm{mg}, 0.048 \mathrm{mmol}, 4$ equiv) was added to $\mathrm{MeOH}(10 \mathrm{~mL})$ and degassed for $20 \mathrm{~min}$. Gold sensors with SAM were removed from the initiator solution, washed with $\mathrm{EtOH}$, dried, and placed in a Schlenk flask. The light blue reaction solution was transferred to this flask via cannula. To initiate the polymerization, $2 \mathrm{~mL}$ of ascorbic acid solution was added. The reaction was then shaken $(50 \mathrm{rpm})$, and an additional $1 \mu \mathrm{L} \mathrm{min}{ }^{-1}$ ascorbic acid solution was continuously supplied via a syringe pump and a PTFE tube until the reaction was quenched by exposure to air. Finally, the sensors were rinsed with Milli-Q water and EtOH and dried.

Measurements. Details of IR-RAS, QCMD, SPR, and nanoplasmonic measurements can be found in the Supporting Information.

\section{RESULTS AND DISCUSSION}

PNIPAM-co-MBAM networks were prepared on gold surfaces initiated with an $\omega$-mercaptoundecyl bromoisobutyrate selfassembled monolayer (SAM) via SI-ARGET-ATRP (Scheme 1 ), taking inspiration from previously described procedures. ${ }^{27,30,37-41}$ The chemical composition of networks with varying amounts of crosslinker were confirmed by infrared spectroscopy (Figure S1), which proved in line with the reported spectra. ${ }^{42}$ The appearance and growth of a band at 

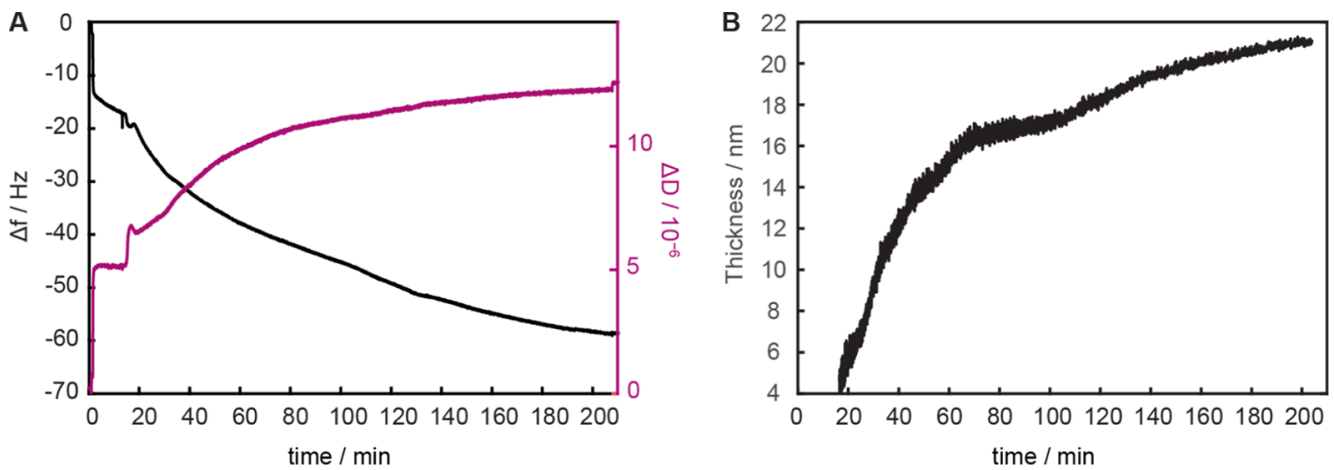

Figure 1. In situ ATRP ( $\mathrm{MeOH}, 0.48 \mathrm{M}$ ) monitored in QCMD yields PNIPAM-co-MBAM $1 \%$. (A) Change in frequency and dissipation over time. (B) Layer thickness over time calculated by Voigt modeling and curve fitting of multiple frequency and dissipation overtones.
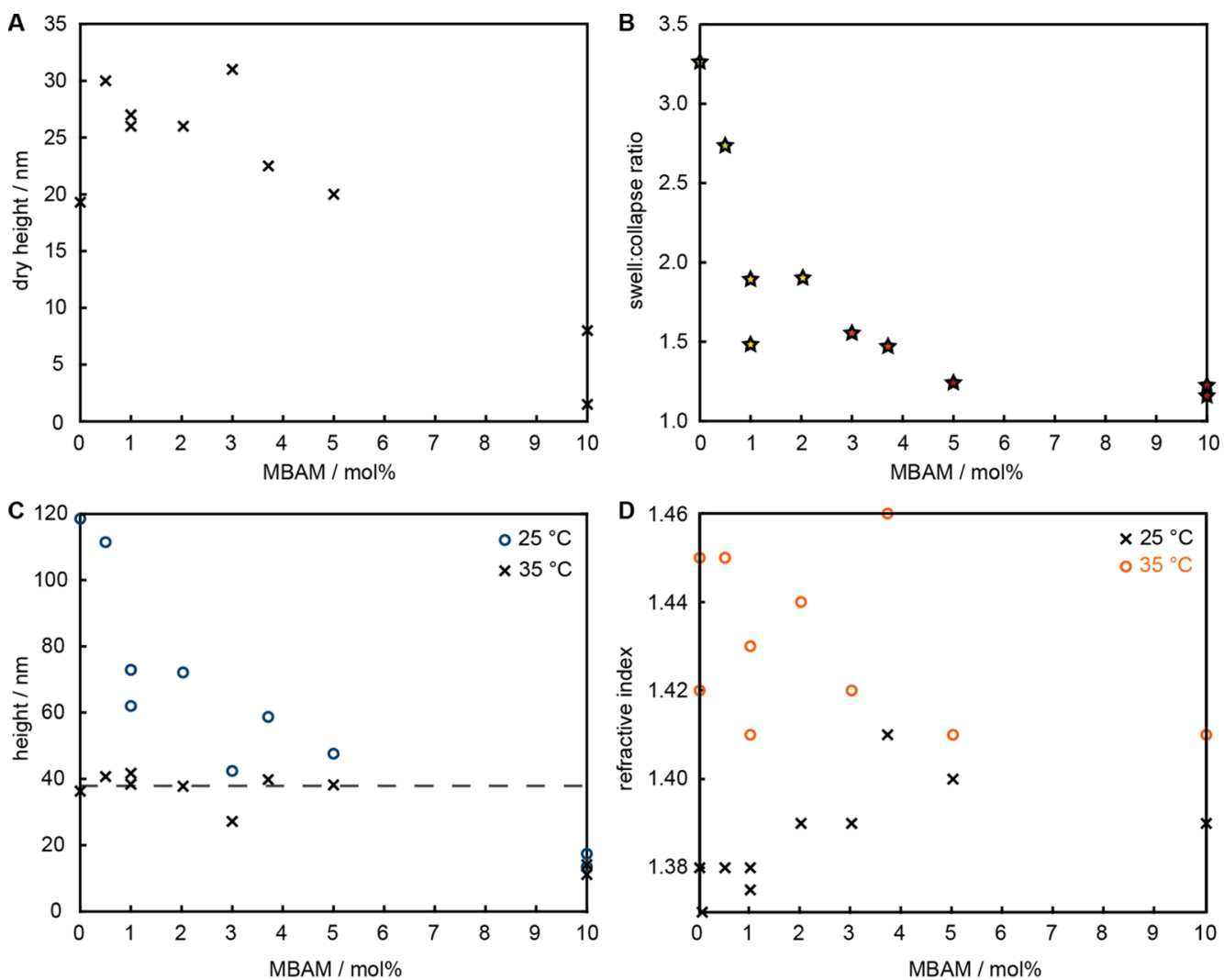

Figure 2. (A) Dry heights of PNIPAM-co-MBAM networks polymerized in $24 \mathrm{~h}(0.96 \mathrm{M}, \mathrm{MeOH})$ decrease with increasing crosslinker content. (B) The swell/collapse height ratio decreases with increasing crosslinker content in the PNIPAM brushes. (C) Decrease in swollen height at $25{ }^{\circ} \mathrm{C}$ and constant collapsed height at $35^{\circ} \mathrm{C}$. (D) Refractive index of PNIPAM-co-MBAM networks with varying crosslinker content in PBS (pH 7.5) at 25 and $35{ }^{\circ} \mathrm{C}$.

$1725 \mathrm{~cm}^{-1}$ with increasing crosslinker content was attributed to a $\mathrm{C}=\mathrm{O}$ stretch from MBAM and confirmed the incorporation of the crosslinker.

To study the kinetics of polymerization, reactions with an NIPAM/MBAM molar ratio of 99:1 (0.96 M) were carried out in methanol for up to $30 \mathrm{~h}$ at ambient temperature. The resulting polymer network heights were measured in air using surface plasmon resonance (SPR). The polymerization proceeded quickly initially: after $1 \mathrm{~h}, 28 \mathrm{~nm}$-thick networks were observed. However, the gel thickness remained constant after this (up to $30 \mathrm{~h}$, Figure S2), presumably due to the significant termination reactions caused by low local monomer concentration, radical combination, or catalyst loss, in line with what has been previously observed. ${ }^{24,34}$ For all reaction times, the SPR angle $\left(\theta_{\mathrm{SPR}}\right)$ and thus the dry height of any particular surface remained constant $\left(\Delta\right.$ dry $\left._{\text {average }}=1.0 \mathrm{~nm}\right)$ across two different positions (Table S2), indicating a homogeneous gel thickness. For better control over thin networks, the ATRP kinetics can be slowed by lowering the monomer concentration. $^{43}$ Thus, an in situ polymerization of PNIPAM-co$\mathrm{MBAM}_{1 \%}$ was monitored at a lower concentration $(0.48 \mathrm{M})$ using QCMD (Figure 1A). The initial decrease in frequency (increase in coupled mass) and simultaneous increase in dissipation occur due to the formation of a viscoelastic layer, that is, polymerization initiation. This was followed by linear growth until $\sim 2 \mathrm{~h}$. The dissipation starts to flatten after $\sim 1 \mathrm{~h}$, indicating the formation of a more rigid layer compared to initially, potentially due to enhanced crosslinking. In general, 


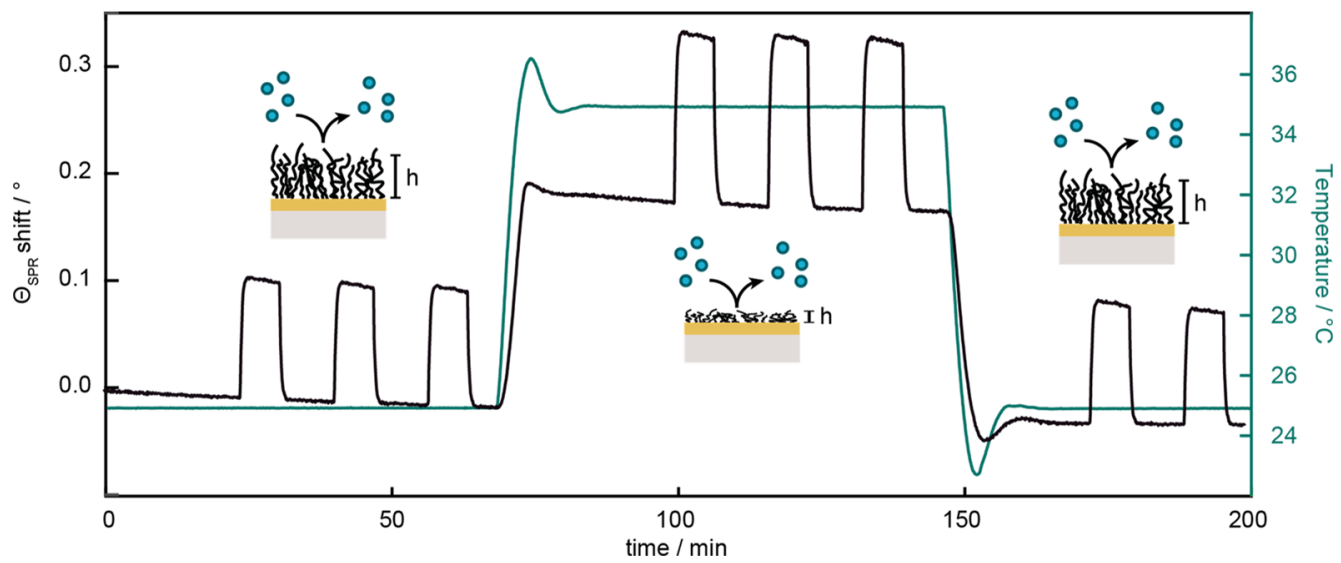

Figure 3. Non-interacting probe method in SPR using PEG $\left(35 \mathrm{kDa}, 10 \mathrm{mg} \mathrm{mL}^{-1}\right)$ at 25 and $35^{\circ} \mathrm{C}$. PEG injections are indicated by reversible $\theta_{\mathrm{SPR}}$ changes of $\sim 0.1^{\circ}$. $\theta_{\mathrm{SPR}}$ increases with rising refractive index caused by a collapsing network at $35^{\circ} \mathrm{C}$ and is fully reversible upon cooling.
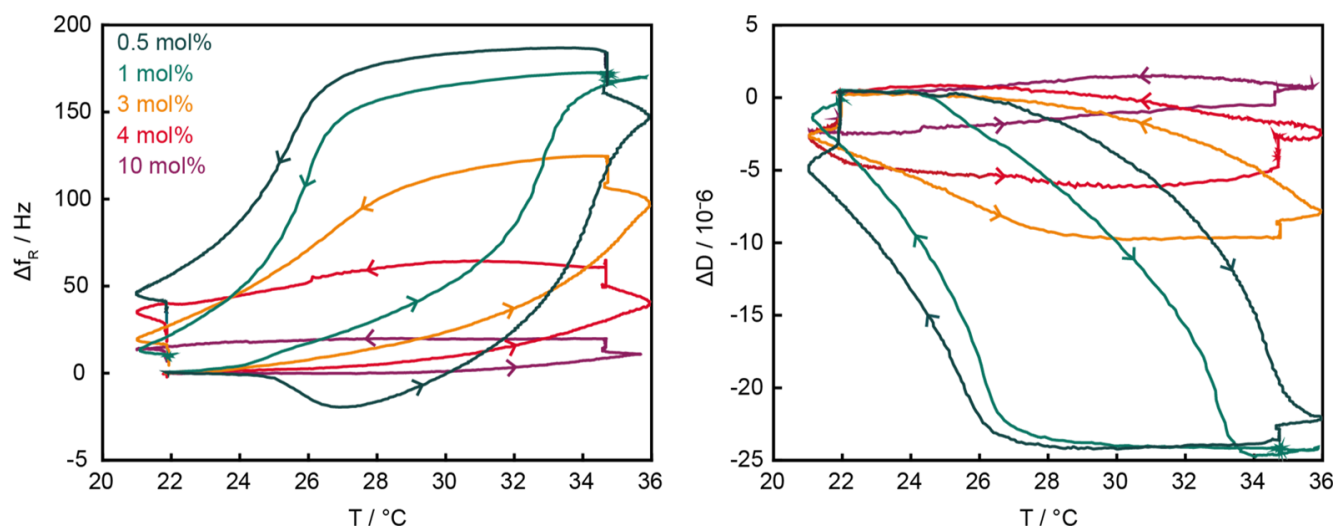

Figure 4. QCMD plot (left: resonance frequency, right: dissipation) of PNIPAM-co-MBAM with 0.5 mol \% (dark green), 1 mol \% (green), 3 mol $\%$ (orange), $4 \mathrm{~mol} \%$ (red), and $10 \mathrm{~mol} \%$ (purple) crosslinker content.

given the relatively low-frequency signals, the flattening curves again point toward termination reactions, similar to more concentrated polymerizations as discussed above. While modeling viscoelastic layers is often challenging, ${ }^{44,45}$ we were able to apply Voigt-based models to quantitatively determine the layer thickness evolution by fitting changes in frequency and dissipation at multiple harmonics (Figure S3). ${ }^{46-49} \mathrm{~A}$ frequency-independent PNIPAM-co-MBAM layer density of $860 \mathrm{~kg} \mathrm{~m}^{-3}$ (20\% polymer brush and $80 \%$ methanol) and standard methanol density and viscosity ${ }^{50}$ were assumed. The maximum gel thickness measured was $21 \mathrm{~nm}$ at $3.5 \mathrm{~h}$ (Figure 1B). This is lower than heights measured by SPR but is not unrealistic as despite measures to maintain an inert atmosphere, some inhibition due to oxygen is expected compared to ex situ polymerizations (e.g., as PTFE tubing is permeable).

Crosslinked brushes with different fractions of MBAM were prepared in polymerizations of $24 \mathrm{~h}$ (the percentage crosslinker indicated is that from the reaction mixture, which we assume represents the percentage in the brush over these reaction durations). With a constant reaction time, the polymer network dry thickness decreased with increasing crosslinker content (Figure 2A). This is not unexpected, as crosslinkers are suspected to increase early termination reactions, ${ }^{24}$ and acrylic monomers can interact with the catalytic CuI/PMDETA complex, hindering ATRP. ${ }^{51}$

Using polyethylene glycol (PEG) $\left(35 \mathrm{kDa}, 10 \mathrm{mg} \mathrm{mL}^{-1}\right)$ as a non-interacting probe in SPR, ${ }^{34}$ we measured "swollen" and "collapsed" exclusion heights, that is, the characteristic height at which the probe molecules are expelled from the brush, of PNIPAM-co-MBAM brushes in PBS at 25 and $35{ }^{\circ} \mathrm{C}$, respectively (Figure 3 ). The linear relation between the total internal reflection angle (which corresponds to bulk effects) and the SPR angle (which responds to both bulk and surface effects) confirms the non-interacting nature of PEG (Figure S4) and indicate that the change in SPR signal only corresponds to changes in the bulk refractive index. ${ }^{44}$ Collapsed gel networks were not free of water but contained roughly $45-50 \%$ by volume, calculated from the ratio of collapsed film thickness (in PBS at $35^{\circ} \mathrm{C}$ ) to dry film thickness in air. The ratio between swollen and collapsed heights, that is, the difference in wet height for the same surface at different temperatures $\left(25\right.$ and $35^{\circ} \mathrm{C}$, respectively), is one important aspect of the thermoresponsive behavior of the PNIPAM networks, and how we define such behavior in this work. As this ratio approaches 1 , thermoresponsive behavior is deemed to be lost. It was not observed to be a function of network height, but instead this ratio gradually decreased with increasing crosslinker content (Figure 2B). This is a direct consequence of decreasing swollen heights in more crosslinked networks as the collapsed heights remain constant (Figure $2 \mathrm{C}$ ). Notably, the thermoresponsive behavior is almost completely lost in networks containing $5 \mathrm{~mol} \%$ or more MBAM (swell/collapse factor $\sim 1.2$ ) in a similar manner to PNIPAM-co-MBAM microgels deposited on solid substrates. ${ }^{36}$ The same trend is observed in the refractive indices: constant 

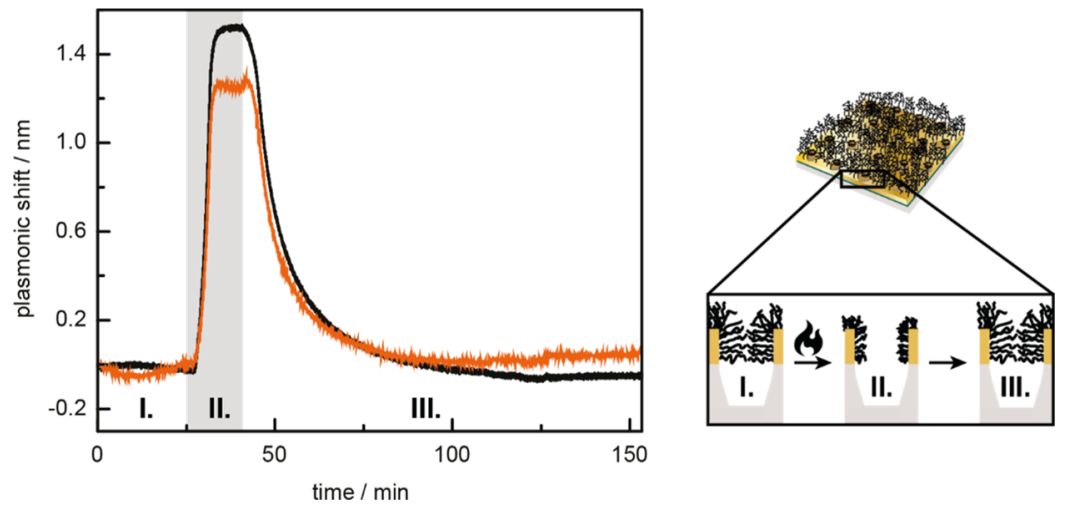

Figure 5. Monitoring shifts in peak (black) and dip (orange) position of a nanowell-patterned sensor coated with PNIPAM-co-MBAM $1 \%$ upon a temperature increase $\left(35^{\circ} \mathrm{C}\right.$, grey background) and subsequent decrease. The collapse and reswelling of the gel layer, opening and closing the pores respectively, is illustrated on the right.

at $35{ }^{\circ} \mathrm{C}$ regardless of MBAM content (supporting that the networks are equally dense in the collapsed state), but increases at $25{ }^{\circ} \mathrm{C}$ in more crosslinked networks (Figure 2D). The lack of thermoresponsive behavior shown here is quite different from that reported for 5\% ene-modified PNIPAM spin-coated and simultaneously crosslinked through thiol-ene click chemistry on a silica substrate (swelling factor $\sim 2.7$ in water). ${ }^{35}$ Grafted-from gel networks are presumably influenced more by crosslinking as the applied shear in the spin-coating process might orient the networks preferentially in one direction ${ }^{28}$ and thereby reduce geometrical constraints that reduce the swollen thickness. Our crosslinked PNIPAMco-MBAM ${ }_{5 \%}$ networks retained some hydrogel characteristic with swollen heights of approximately double the dry heights and $\sim 60$ vol $\%$ water content above the VPTT $\left(35^{\circ} \mathrm{C}\right)$.

The reversible nature of the volume phase transition was confirmed by SPR (Figure 3). Upon expelling water when heating to $35{ }^{\circ} \mathrm{C}$, the refractive index of the layer close to the surface increased, simultaneously causing a shift in $\theta_{\mathrm{SPR}} \cdot{ }^{34}$ Cooling back to $25{ }^{\circ} \mathrm{C}$ resulted in a reswelling of the gel to the same extent as before the first collapse, indicated by a shift in $\theta_{\text {SPR }}$ back to the original position.

Integrated real-time temperature output with QCMD revealed further details on the temperature range in which the phase transition occurs. Upon heating to $35{ }^{\circ} \mathrm{C}$, the networks expel water (i.e., lose mass) and the resonance frequency increases. Simultaneously, the layer rigidifies, as evident by a decrease in dissipation (Figure 4). The phase transition is less pronounced in networks with a higher crosslinker content and is nonexistent in gels containing 10 mol \% MBAM. The collapse takes place in a broad temperature regime between 27 and $34{ }^{\circ} \mathrm{C}$ for all crosslinker contents investigated (Figures 4 and S5). A different trend is visible in the reswelling of the layers upon cooling back to 22 ${ }^{\circ} \mathrm{C}$ : for networks containing up to $1 \mathrm{~mol} \% \mathrm{MBAM}$, frequency and dissipation remained constant until $26^{\circ} \mathrm{C}$, followed by a sharp change within $2{ }^{\circ} \mathrm{C}$, whereas more crosslinked layers transitioned gently within a $6{ }^{\circ} \mathrm{C}$ range (between 28 and 22 ${ }^{\circ} \mathrm{C}$ ). Previously, differences between swelling and collapsing cycles were attributed to a "conformational memory effect": inter- and intramolecular H-bonding in the collapsed state which suppresses rehydration. ${ }^{52,53}$ Crosslinking generally hinders hydration of surface-attached networks; ${ }^{23-25}$ thus, the gentle reswelling in our more crosslinked networks could result from a combination of a more pronounced memory effect and a slight VPTT increase in more crosslinked networks. ${ }^{27}$ Cycling between collapse and reswelling more slowly than the current rate $\left(\sim 0.3{ }^{\circ} \mathrm{C} \mathrm{s}^{-1}\right)$ would likely decrease this hysteresis. ${ }^{34}$ Again, these results differ from the spin-coated PNIPAM gels (5\% crosslinker) for which a sharp transition between 33 and $36{ }^{\circ} \mathrm{C}$ was observed. ${ }^{35}$

Looking toward applications on nanostructured surfaces, ${ }^{54}$ we studied the behavior of PNIPAM-co-MBAM thermoresponsive gels on thin gold films containing plasmonic nanowell arrays (diameter: $90 \mathrm{~nm}$ ) with optical properties discussed in detail in a previous report by our group. ${ }^{55}$ Briefly, such plasmonic structures display characteristic extinction spectra (absorption + scattering) with resonance features originating from the apertures in the thin metal film. Shifts in peak and dip position of the asymmetric resonance (Figure S6) correspond to refractive index changes on the surface and inside the nanowells, respectively. ${ }^{55,56}$ The thermoresponsive swelling behavior of our gel networks was retained on the patterned surfaces and mirrored that from the planar surfaces: more crosslinked networks swelled less (Figures 5 and S7). Thus, we propose that PNIPAM-co-MBAM brush networks are suitable for implementation on porous supports. They display tunable thermoresponsive behavior when surface confined and provide a logical next step beyond the PNIPAM brushes that have been investigated previously inside porous membranes. ${ }^{57,58}$

\section{CONCLUSIONS}

In conclusion, PNIPAM-co-MBAM networks were synthesized via a simple and reliable SI-ARGET-ATRP strategy on gold films with thiol SAMs. At $0.96 \mathrm{M}$, the polymerization proceeded with fast reaction kinetics, giving networks with dry thicknesses $>25 \mathrm{~nm}$ within $1 \mathrm{~h}$. For better control over networks, the ATRP could be slowed by lowering the reaction concentration as demonstrated by an in situ QCMD experiment. The thermoresponsive phase transition of PNIPAM-coMBAM gels is characterized by the swollen/collapsed height ratio. The ratio was not dependent on network height but could be tuned by varying the crosslinker content between 0 and $10 \mathrm{~mol} \%$. Investigations in SPR and QCMD showed that swollen gel heights (below VPTT) decreased with increasing crosslinker content, while collapsed heights (above VPTT) remained constant. This caused loss of thermoresponsiveness in gels containing at least $5 \mathrm{~mol} \% \mathrm{MBAM}$, a significantly different behavior than observed in previous studies on spincoated or bulk PNIPAM-based networks. ${ }^{28,35,36,59}$ A broader 
transition range was also evident for more crosslinked networks. In all, we have demonstrated a reliable method for tuning the swelling behavior of PNIPAM networks grafted from planar surfaces and then expanded this to patterned thin gold films containing nanowell arrays. By studying the behavior of PNIPAM-co-MBAM gels on nanostructured surfaces, we have paved the way for expanding their scope as thermoresponsive devices.

\section{ASSOCIATED CONTENT}

\section{SI Supporting Information}

The Supporting Information is available free of charge at https://pubs.acs.org/doi/10.1021/acs.langmuir.0c03545.

Method descriptions and spectra of IR-RAS, QCMD (with in situ polymerization), SPR, and LSPR; polymerization kinetic plot; SPR fitting parameters; film thicknesses obtained from SPR measurements; and estimated network water content (PDF)

\section{AUTHOR INFORMATION}

\section{Corresponding Author}

Rebekah L. N. Hailes - Department of Chemistry and Chemical Engineering, Chalmers University of Technology, 41296 Gothenburg, Sweden; (1) orcid.org/0000-0001-88956426; Email: rebekah@chalmers.se

\section{Authors}

Sophia Thiele - Department of Chemistry and Chemical Engineering, Chalmers University of Technology, 41296 Gothenburg, Sweden

John Andersson - Department of Chemistry and Chemical Engineering, Chalmers University of Technology, 41296 Gothenburg, Sweden; (1) orcid.org/0000-0002-2977-8305

Andreas Dahlin - Department of Chemistry and Chemical Engineering, Chalmers University of Technology, 41296 Gothenburg, Sweden

Complete contact information is available at: https://pubs.acs.org/10.1021/acs.langmuir.0c03545

\section{Author Contributions}

The manuscript was written through contributions of all authors. All authors have given approval to the final version of the manuscript.

\section{Funding}

This work was financed by the Knut \& Alice Wallenberg Foundation and the Erling-Persson Family Foundation.

Notes

The authors declare no competing financial interest.

\section{ACKNOWLEDGMENTS}

This work was performed in part at the Chalmers Material Analysis Laboratory, CMAL. S.T. gratefully acknowledges Justas Svirelis for useful early stage discussions.

\section{REFERENCES}

(1) Conzatti, G.; Cavalie, S.; Combes, C.; Torrisani, J.; Carrere, N.; Tourrette, A. PNIPAM Grafted Surfaces through ATRP and RAFT Polymerization: Chemistry and Bioadhesion. Colloids Surf., B 2017, 151, 143-155.

(2) Doberenz, F.; Zeng, K.; Willems, C.; Zhang, K.; Groth, T. Thermoresponsive polymers and their biomedical application in tissue engineering - a review. J. Mater. Chem. B 2020, 8, 607-628.
(3) Oh, J. K.; Drumright, R.; Siegwart, D. J.; Matyjaszewski, K. The Development of Microgels/Nanogels for Drug Delivery Applications. Prog. Polym. Sci. 2008, 33, 448-477.

(4) Schmidt, S.; Zeiser, M.; Hellweg, T.; Duschl, C.; Fery, A.; Möhwald, H. Adhesion and Mechanical Properties of PNIPAM Microgel Films and Their Potential Use as Switchable Cell Culture Substrates. Adv. Funct. Mater. 2010, 20, 3235-3243.

(5) Tian, Y.; Wei, X.; Wang, Z. J.; Pan, P.; Li, F.; Ling, D.; Wu, Z. L.; Zheng, Q. A Facile Approach to Prepare Tough and Responsive Ultrathin Physical Hydrogel Films as Artificial Muscles. ACS Appl. Mater. Interfaces 2017, 9, 34349-34355.

(6) Bojko, A.; Andreatta, G.; Montagne, F.; Renaud, P.; Pugin, R. Fabrication of Thermo-Responsive Nano-Valve by Grafting-to in Melt of Poly(N-Isopropylacrylamide) onto Nanoporous Silicon Nitride Membranes. J. Membr. Sci. 2014, 468, 118-125.

(7) Zhang, H.; Cooper, A. I. Thermoresponsive "Particle Pumps": Activated Release of Organic Nanoparticles from Open-Cell Macroporous Polymers. Adv. Mater. 2007, 19, 2439-2444.

(8) Chen, T.; Ferris, R.; Zhang, J.; Ducker, R.; Zauscher, S. StimulusResponsive Polymer Brushes on Surfaces: Transduction Mechanisms and Applications. Prog. Polym. Sci. 2010, 35, 94-112.

(9) Stuart, M. A. C.; Huck, W. T. S.; Genzer, J.; Müller, M.; Ober, C.; Stamm, M.; Sukhorukov, G. B.; Szleifer, I.; Tsukruk, V. V.; Urban, M.; Winnik, F.; Zauscher, S.; Luzinov, I.; Minko, S. Emerging Applications of Stimuli-Responsive Polymer Materials. Nat. Mater. 2010, 9, 101-113.

(10) Gerges, G. G.; Irini, M. M.; Blasi, B.; Argentiere, S. Smart Microfluidics: The Role of Stimuli-Responsive Polymers in Microfluidic Devices. In Advances in Microfluidics; Kelly, R. T., Ed.; IntechOpen: InTech, 2012; pp 127-154.

(11) Plunkett, K. N.; Zhu, X.; Moore, J. S.; Leckband, D. E. PNIPAM Chain Collapse Depends on the Molecular Weight and Grafting Density. Langmuir 2006, 22, 4259-4266.

(12) Malham, I. B.; Bureau, L. Density Effects on Collapse, Compression, and Adhesion of Thermoresponsive Polymer Brushes. Langmuir 2010, 26, 4762-4768.

(13) Ejaz, M.; Tsujii, Y.; Fukuda, T. Controlled Grafting of a WellDefined Polymer on a Porous Glass Filter by Surface-Initiated Atom Transfer Radical Polymerization. Polymer 2001, 42, 6811-6815.

(14) Bao, Z.; Bruening, M. L.; Baker, G. L. Control of the Density of Polymer Brushes Prepared by Surface-Initiated Atom Transfer Radical Polymerization. Macromolecules 2006, 39, 5251-5258.

(15) Behling, R. E.; Williams, B. A.; Staade, B. L.; Wolf, L. M.; Cochran, E. W. Influence of Graft Density on Kinetics of SurfaceInitiated Atrp of Polystyrene from Montmorillonite. Macromolecules 2009, 42, 1867-1872.

(16) Wei, J.; He, P.; Liu, A.; Chen, X.; Wang, X.; Jing, X. Surface Modification of Hydroxyapatite Nanoparticles with ThermalResponsive PNIPAM by ATRP. Macromol. Biosci. 2009, 9, 12371246.

(17) Wu, T.; Zhang, Y.; Wang, X.; Liu, S. Fabrication of Hybrid Silica Nanoparticles Densely Grafted with Thermoresponsive Poly $(\mathrm{N}$ Isopropylacrylamide) Brushes of Controlled Thickness via SurfaceInitiated Atom Transfer Radical Polymerization. Chem. Mater. 2008, 20, 101-109.

(18) Jones, D. M.; Smith, J. R.; Huck, W. T. S.; Alexander, C. Variable Adhesion of Micropatterned Thermoresponsive Polymer Brushes: AFM Investigations of Poly(N-Isopropylacrylamide) Brushes Prepared by Surface-Initiated Polymerizations. Adv. Mater. 2002, 14, 1130-1134.

(19) Tu, H.; Heitzman, C. E.; Braun, P. V. Patterned Poly(NIsopropylacrylamide) Brushes on Silica Surfaces by Microcontact Printing Followed by Surface-Initiated Polymerization. Langmuir 2004, 20, 8313-8320.

(20) Khabibullin, A.; Mastan, E.; Matyjaszewski, K.; Zhu, S. SurfaceInitiated Atom Transfer Radical Polymerization. In Controlled Radical Polymerization at and from Solid Surfaces; Vana, P., Ed.; Springer International Publishing: Cham, 2015; Vol. 270. 
(21) Zoppe, J. O.; Ataman, N. C.; Mocny, P.; Wang, J.; Moraes, J.; Klok, H.-A. Surface-Initiated Controlled Radical Polymerization: State-of-the-Art, Opportunities, and Challenges in Surface and Interface Engineering with Polymer Brushes. Chem. Rev. 2017, 117, $1105-1318$

(22) Yim, H.; Kent, M. S.; Mendez, S.; Lopez, G. P.; Satija, S.; Seo, Y. Effects of Grafting Density and Molecular Weight on the Temperature-Dependent Conformational Change of Poly(N-Isopropylacrylamide) Grafted Chains in Water. Macromolecules 2006, 39, 3420-3426.

(23) Wong, R.; Ashton, M.; Dodou, K. Effect of Crosslinking Agent Concentration on the Properties of Unmedicated Hydrogels. Pharmaceutics 2015, 7, 305-319.

(24) Lilge, I.; Schönherr, H. Covalently Cross-Linked Poly(Acrylamide) Brushes on Gold with Tunable Mechanical Properties via Surface-Initiated Atom Transfer Radical Polymerization. Eur. Polym. J. 2013, 49, 1943-1951.

(25) Li, A.; Benetti, E. M.; Tranchida, D.; Clasohm, J. N.; Schönherr, H.; Spencer, N. D. Surface-Grafted, Covalently Cross-Linked Hydrogel Brushes with Tunable Interfacial and Bulk Properties. Macromolecules 2011, 44, 5344-5351.

(26) Dehghani, E. S.; Ramakrishna, S. N.; Spencer, N. D.; Benetti, E. M. Controlled Crosslinking Is a Tool To Precisely Modulate the Nanomechanical and Nanotribological Properties of Polymer Brushes. Macromolecules 2017, 50, 2932-2941.

(27) Li, D.; He, Q.; Cui, Y.; Wang, K.; Zhang, X.; Li, J. Thermosensitive Copolymer Networks Modify Gold Nanoparticles for Nanocomposite Entrapment. Chem.-Eur. J. 2007, 13, 22242229.

(28) Harmon, M. E.; Kuckling, D.; Pareek, P.; Frank, C. W. PhotoCross-Linkable PNIPAAm Copolymers. 4. Effects of Copolymerization and Cross-Linking on the Volume-Phase Transition in Constrained Hydrogel Layers. Langmuir 2003, 19, 10947-10956.

(29) Sanson, N.; Rieger, J. Synthesis of Nanogels/Microgels by Conventional and Controlled Radical Crosslinking Copolymerization. Polym. Chem. 2010, 1, 965-977.

(30) Matyjaszewski, K.; Dong, H.; Jakubowski, W.; Pietrasik, J.; Kusumo, A. Grafting from Surfaces for "Everyone": ARGET ATRP in the Presence of Air. Langmuir 2007, 23, 4528-4531.

(31) Yeow, J.; Chapman, R.; Gormley, A. J.; Boyer, C. Up in the Air: Oxygen Tolerance in Controlled/Living Radical Polymerisation. Chem. Soc. Rev. 2018, 47, 4357-4387.

(32) Halperin, A.; Zhulina, E. B. Atomic Force Microscopy of Polymer Brushes: Colloidal versus Sharp Tips. Langmuir 2010, 26, 8933-8940.

(33) Parisse, P.; Solano, I.; Magnozzi, M.; Bisio, F.; Casalis, L.; Cavalleri, O.; Canepa, M. Thickness and Beyond. Exploiting Sepctroscopic Ellipsometry and Atomic Force Nanolithography for the Investigation of Ultrathin Interfaces of Biologic Interest. In Ellipsometry of Functional Organic Surfaces and Films; Hinrichts, K., Eichhorn, K.-J., Eds.; Springer International Publishing: Cham, 2018; pp 63-93.

(34) Emilsson, G.; Schoch, R. L.; Oertle, P.; Xiong, K.; Lim, R. Y. H.; Dahlin, A. B. Surface plasmon resonance methodology for monitoring polymerization kinetics and morphology changes of brushes-evaluated with poly(N-isopropylacrylamide). Appl. Surf. Sci. 2017, 396, 384-392.

(35) Li, M.; Bresson, B.; Cousin, F.; Fretigny, C.; Tran, Y. Submicrometric Films of Surface-Attached Polymer Network with Temperature-Responsive Properties. Langmuir 2015, 31, 1151611524

(36) Schulte, M. F.; Scotti, A.; Gelissen, A. P. H.; Richtering, W.; Mourran, A. Probing the Internal Heterogeneity of Responsive Microgels Adsorbed to an Interface by a Sharp SFM Tip: Comparing Core-Shell and Hollow Microgels. Langmuir 2018, 34, 4150-4158.

(37) Mendonça, P. V.; Averick, S. E.; Konkolewicz, D.; Serra, A. C.; Popov, A. V.; Guliashvili, T.; Matyjaszewski, K.; Coelho, J. F. J. Straightforward ARGET ATRP for the Synthesis of Primary Amine
Polymethacrylate with Improved Chain-End Functionality under Mild Reaction Conditions. Macromolecules 2014, 47, 4615-4621.

(38) Wang, Y.; Soerensen, N.; Zhong, M.; Schroeder, H.; Buback, M.; Matyjaszewski, K. Improving the "Livingness" of ATRP by Reducing Cu Catalyst Concentration. Macromolecules 2013, 46, 683691.

(39) Nanda, A. K.; Matyjaszewski, K. Effect of [PMDETA]/[Cu(I)] Ratio, Monomer, Solvent, Counterion, Ligand, and Alkyl Bromide on the Activation Rate Constants in Atom Transfer Radical Polymerization. Macromolecules 2003, 36, 1487-1493.

(40) Min, K.; Gao, H.; Matyjaszewski, K. Use of Ascorbic Acid as Reducing Agent for Synthesis of Well-Defined Polymers by ARGET ATRP. Macromolecules 2007, 40, 1789-1791.

(41) Horn, M.; Matyjaszewski, K. Solvent Effects on the Activation Rate Constant in Atom Transfer Radical Polymerization. Macromolecules 2013, 46, 3350-3357.

(42) Sun, B.; Lin, Y.; Wu, P. Structure Analysis of Poly(NIsopropylacrylamide) Using Near-Infrared Spectroscopy and Generalized Two-Dimensional Correlation Infrared Spectroscopy. Appl. Spectrosc. 2007, 61, 765-771.

(43) Matyjaszewski, K. Atom Transfer Radical Polymerization (ATRP): Current Status and Future Perspectives. Macromolecules 2012, 45, 4015-4039.

(44) Ferrand-Drake Del Castillo, G.; Emilsson, G.; Dahlin, A. Quantitative Analysis of Thickness and PH Actuation of Weak Polyelectrolyte Brushes. J. Phys. Chem. C 2018, 122, 27516-27527.

(45) Dunér, G.; Thormann, E.; Dédinaite, A. Quartz Crystal Microbalance with Dissipation (QCM-D) Studies of the Viscoelastic Response from a Continuously Growing Grafted Polyelectrolyte Layer. J. Colloid Interface Sci. 2013, 408, 229-234.

(46) Voinova, M. V.; Rodahl, M.; Jonson, M.; Kasemo, B. Viscoelastic Acoustic Response of Layered Polymer Films at FluidSolid Interfaces: Continuum Mechanics Approach. Phys. Scr. 1999, 59, 391-396.

(47) Höök, F.; Kasemo, B.; Nylander, T.; Fant, C.; Sott, K.; Elwing, H. Variations in Coupled Water, Viscoelastic Properties, and Film Thickness of a Mefp-1 Protein Film during Adsorption and CrossLinking: A Quartz Crystal Microbalance with Dissipation Monitoring, Ellipsometry, and Surface Plasmon Resonance Study. Anal. Chem. 2001, 73, 5796-5804.

(48) Reviakine, I.; Johannsmann, D.; Richter, R. P. Hearing What You Cannot See and Visualizing What You Hear: Interpreting Quartz Crystal Microbalance Data from Solvated Interfaces. Anal. Chem. 2011, 83, 8838-8848.

(49) Saftics, A.; Prósz, G. A.; Türk, B.; Peter, B.; Kurunczi, S.; Horvath, R. In Situ Viscoelastic Properties and Chain Conformations of Heavily Hydrated Carboxymethyl Dextran Layers: A Comparative Study Using OWLS and QCM-I Chips Coated with Waveguide Material. Sci. Rep. 2018, 8, 11840.

(50) Mikhail, S. Z.; Kimel, W. R. Densities and Viscosities of Methanol-Water Mixtures. J. Chem. Eng. Data 1961, 6, 533-537.

(51) Braunecker, W. A.; Tsarevsky, N. V.; Pintauer, T.; Gil, R. R.; Matyjaszewski, K. Quantifying Vinyl Monomer Coordination to Culin Solution and the Effect of Coordination on Monomer Reactivity in Radical Copolymerization. Macromolecules 2005, 38, 4081-4088.

(52) Tokarev, I.; Minko, S. Stimuli-Responsive Hydrogel Thin Films. Soft Matter 2009, 5, 511-524.

(53) Cheng, H.; Shen, L.; Wu, C. LLS and FTIR Studies on the Hysteresis in Association and Dissociation of Poly(N-Isopropylacrylamide) Chains in Water. Macromolecules 2006, 39, 2325-2329.

(54) Sharma, N.; Keshmiri, H.; Zhou, X.; Wong, T. I.; Petri, C.; Jonas, U.; Liedberg, B.; Dostalek, J. Tunable Plasmonic Nanohole Arrays Actuated by a Thermoresponsive Hydrogel Cushion. J. Phys. Chem. C 2016, 120, 561-568.

(55) Malekian, B.; Xiong, K.; Emilsson, G.; Andersson, J.; Fager, C.; Olsson, E.; Larsson-Langhammer, E.; Dahlin, A. Fabrication and Characterization of Plasmonic Nanopores with Cavities in the Solid Support. Sensors 2017, 17, 1444. 
(56) Junesch, J.; Emilsson, G.; Xiong, K.; Kumar, S.; Sannomiya, T.; Pace, H.; Vörös, J.; Oh, S.-H.; Bally, M.; Dahlin, A. B. LocationSpecific Nanoplasmonic Sensing of Biomolecular Binding to Lipid Membranes with Negative Curvature. Nanoscale 2015, 7, 1508015085 .

(57) Alem, H.; Duwez, A.-S.; Lussis, P.; Lipnik, P.; Jonas, A. M.; Demoustier-Champagne, S. Microstructure and Thermo-Responsive Behavior of Poly(N-Isopropylacrylamide) Brushes Grafted in Nanopores of Track-Etched Membranes. J. Membr. Sci. 2008, 308, 75-86. (58) Chu, L.-Y.; Niitsuma, T.; Yamaguchi, T.; Nakao, S.-I. Thermoresponsive Transport through Porous Membranes with Grafted PNIPAM Gates. AIChE J. 2003, 49, 896-909.

(59) Pong, F. Y.; Lee, M.; Bell, J. R.; Flynn, N. T. Thermoresponsive Behavior of Poly(N-Isopropylacrylamide) Hydrogels Containing Gold Nanostructures. Langmuir 2006, 22, 3851-3857. 